PROCEEDINGS OF THE

AMERICAN MATHEMATICAL SOCIETY

Volume 133, Number 5 , Pages 1451-1458

S 0002-9939(04)07695-6

Article electronically published on November 22, 2004

\title{
SOME REMARKS ON AN EXISTENCE PROBLEM FOR DEGENERATE ELLIPTIC SYSTEMS
}

\author{
OLLI MARTIO, VLADIMIR MIKLYUKOV, AND MATTI VUORINEN
}

(Communicated by Richard A. Wentworth)

\begin{abstract}
The system $a u_{x}+b u_{y}=v_{y}, \quad c u_{x}+d u_{y}=-v_{x}$, which yields Beltrami's system if $b=c$, is considered. Under a condition for the coefficients $a, b, c, d$ a non-existence theorem is proved.
\end{abstract}

\section{MAIN RESUlts}

Let $D, \mathcal{D} \subset \mathbf{R}^{2}$ be simply connected domains of the $z=(x, y)$ and the $w=(u, v)$ planes, respectively. Below we consider the problem of existence of homeomorphic $A C L_{\text {loc }}^{2}$ mappings $w=w(z)$ from $D$ onto $\mathcal{D}$ satisfying the system

$$
a u_{x}+b u_{y}=v_{y}, \quad c u_{x}+d u_{y}=-v_{x} .
$$

A function $h$ is, by definition, in the class $A C L_{\text {loc }}^{2}$ iff $h$ is absolutely continuous along a.e. horizontal and vertical lines and its partial derivatives $h_{x}, h_{y}$ belong to $L_{\text {loc }}^{2}$. Here the coefficients $a, b, c, d$ are measurable functions in $D$. We set

$$
\delta \equiv a d-(b+c)^{2} / 4 .
$$

In what follows we assume that $a>0$ and

$$
\operatorname{ess}_{\inf _{D^{\prime}}}(\delta)>0 \text { for every } D^{\prime} \subset \subset D \text {. }
$$

In the particular case $a=d=1$ and $b=c=0,(1.1)$ is the classic CauchyRiemann system and the solution of the existence problem is given by the Riemann mapping theorem. In the case $b=c$, we have the well-known Beltrami system. For more on the existence theorems given here see, for example, G. David [5], M.A. Brakalova and J.A. Jenkins 2], U. Srebro and E. Yakubov [14, V. Gutlyanskii, O. Martio, T. Sugawa and M. Vuorinen [8], O. Martio and V. M. Miklyukov [10].

In the general case, the global mapping problem is much more complicated than in the aforementioned particular cases. Under the assumption

$$
\operatorname{ess} \inf _{D} \delta>0,
$$

the existence problem was solved by B. Bojarski 1]. If this condition is not fulfilled, then there are isolated results only. The case in which (1.3) is violated at a finite number of boundary points was considered by A. Džuraev [6] and E.A. Chtcherbakov 44. On the other hand, I.S. Ovchinnikov 13 and A.P. Mihailov [12] proved some results pertaining to the solvability problem of the system

Received by the editors June 1, 2003 and, in revised form, January 22, 2004.

2000 Mathematics Subject Classification. Primary 30C62; Secondary 35J70.

(C) 2004 American Mathematical Society Reverts to public domain 28 years from publication 
(1.1) with (1.2) under some special conditions, which allow degeneration close to a boundary arc.

Here we give a condition for the coefficients of (1.1) under which the coordinate function $u(z)$ of $w(z)$ is monotone close to the boundary [11]. We use this condition for a non-existence theorem.

Let $D \subset \mathbf{R}^{2}$ be a domain. By $\tilde{\partial} D$ we denote the boundary of $D$ in the extended plane $\tilde{\mathbf{R}}^{2}=\mathbf{R}^{2} \cup\{\infty\}$. For an arbitrary subdomain $\Delta \subset D$, we set

$$
\partial^{\prime} \Delta=\tilde{\partial} \Delta \backslash \tilde{\partial} D \quad \text { and } \quad \partial^{\prime \prime} \Delta=\tilde{\partial} \Delta \cap \tilde{\partial} D .
$$

Let $\Gamma$ be a subset of $\tilde{\partial} D$. A continuous function $f: D \rightarrow \mathbf{R}$ is called monotone close to $\Gamma$ if for every subdomain $\Delta \subset D$ with $\partial^{\prime \prime} \Delta \subset \Gamma$,

$$
\operatorname{osc}(f, \Delta) \leq \operatorname{osc}\left(f, \partial^{\prime} \Delta\right)
$$

Here the symbol osc $(f, E)$ means the oscillation of $f$ along the set $E$.

Every function $f$ monotone close to $\Gamma$ is monotone in the classical sense of Lebesgue since for domains $\Delta \subset \subset D$, (1.4) reduces to osc $(f, \Delta) \leq \operatorname{osc}(f, \partial \Delta)$. On the other hand, if $\Gamma=\tilde{\partial} D$, then every function monotone close to $\Gamma$ is a constant function. This is obvious since choosing $\Delta=D \backslash\left\{z_{0}\right\}$, where $z_{0} \in D$ is an arbitrary point, by (1.4) we obtain

$$
\operatorname{osc}(f, \Delta) \leq \operatorname{osc}\left(f,\left\{z_{0}\right\}\right)=0 .
$$

We shall describe the behaviour of the coefficients of the system close to a set of degeneracy by means of a special exhaustion function $h$. Fix a set $\Gamma \subset \tilde{\partial} D$ and a positive locally Lipschitz function $h: D \rightarrow \mathbf{R}$ such that $\lim _{z \rightarrow \Gamma} h(z)=0$ and

$$
0<h_{0} \leq \operatorname{essinf}_{D}|\nabla h(z)| \leq \operatorname{ess} \sup _{D}|\nabla h(z)| \leq h_{1}<\infty,
$$

where $h_{0}, h_{1}$ are some constants.

Below we let $E_{t}=\{z \in D: h(z)=t\}$ denote the level curve of $h$.

Theorem 1.6. Let $\Gamma \subset \tilde{\partial} D$ be an arbitrary set, and let $w=(u, v)$ be an $A C L_{\mathrm{loc}}^{2}$ homeomorphic solution of (1.1) from $D$ onto $\mathcal{D}$ satisfying (1.2) such that $|u|<M$ in D. If

$$
\int_{0} d t\left(\int_{D \cap E_{t}}(a+d) \frac{a d-b c}{\delta} d \mathcal{H}^{1}\left(E_{t}\right)\right)^{-1}=\infty
$$

then $u$ is monotone close to $\Gamma$.

For a homeomorphism $w: D \rightarrow \mathcal{D}$ and for an arbitrary $\Gamma \subset \tilde{\partial} D$ we set

$$
w(\Gamma)=\left\{y \in \tilde{\partial} \mathcal{D}: \exists \text { a sequence } z_{n} \in D, z_{n} \rightarrow \Gamma \text {, such that } w\left(z_{n}\right) \rightarrow y\right\} .
$$

Clearly, if $\Gamma \subset \partial D$ is connected and $\partial D$ is a simple Jordan curve, then $w(\Gamma)$ is also connected.

A set $L \subset \tilde{\partial} \mathcal{D}$ is called $u$-forked if there are at least two points $w^{\prime}=\left(u^{\prime}, v^{\prime}\right), w^{\prime \prime}=$ $\left(u^{\prime}, v^{\prime \prime}\right) \in L$ such that the segment

$$
l=\left\{(u, v) \in \mathbf{R}^{2}: u=u^{\prime}, v^{\prime}<v<v^{\prime \prime}\right\} \quad \text { lies in } \mathcal{D}
$$

and separates from $\tilde{\partial} \mathcal{D} \backslash L$ some subdomain $U \subset \mathcal{D}$ with $\tilde{\partial} U \subset \bar{l} \cup L$.

For example, let $\mathcal{D}$ be a disk and let $\partial \mathcal{D}$ be its boundary circle. Then the right and left semicircles are $u$-forked; however, the upper and lower semicircles are not $u$-forked. 
Theorem 1.8. Let $M>0$ be a constant. Suppose that $\mathcal{D}$ is a subdomain of $\{(u, v):|u|<M\}$, the coefficients of (1.1) satisfy (1.7) and $L \subset \tilde{\partial} \mathcal{D}$ is u-forked. Then there is no $A C L_{\mathrm{loc}}^{2}$ homeomorphic solution $w=w(z)$ of (1.1) from $D$ onto $\mathcal{D}$ such that $w(\Gamma) \supset L$.

\section{Proof of Theorem 1.6}

Let $\Delta$ be a subdomain of $D$ with $\partial^{\prime \prime} \Delta \subset \Gamma$. We shall prove that

$$
\sup _{\Delta} u(z)=\sup _{\partial^{\prime} \Delta} u(z)
$$

Assume the contrary, that is, there exists a point $z_{0} \in \Delta$ such that

$$
u\left(z_{0}\right)>\sup _{\partial^{\prime} \Delta} u(z)=A .
$$

Choose $\epsilon>A$ such that $u\left(z_{0}\right)>\epsilon$. Fix the connected component $U$ of the set $\{z \in \Delta: u(z)>\epsilon\}$ containing $z_{0}$. By [15, Theorem 5.4.4] for almost all $\epsilon>A$, the sets $\{z \in \Delta: u(z)=\epsilon\}$ are locally rectifiable. Therefore, without loss of generality, we may assume that $\partial^{\prime} U$ is locally rectifiable.

Fix numbers $0<\delta^{\prime}<\delta^{\prime \prime}<h\left(z_{0}\right)$ and an absolutely continuous function $\psi_{0}$ : $\left[\delta^{\prime}, \delta^{\prime \prime}\right] \rightarrow[0,1]$ such that $\psi_{0}\left(\delta^{\prime \prime}\right)=1$ and $\psi_{0}\left(\delta^{\prime}\right)=0$. We shall specify $\psi_{0}$ later. Define $\psi:(0, \infty) \rightarrow \mathbf{R}$ as

$$
\psi(\tau)= \begin{cases}1 & \text { for } \quad \delta^{\prime \prime}<\tau<\infty \\ \psi_{0}(\tau) & \text { for } \quad \delta^{\prime} \leq \tau \leq \delta^{\prime \prime} \\ 0 & \text { for } \quad 0<\tau<\delta^{\prime}\end{cases}
$$

Then $\psi$ is an absolutely continuous function. Write $\phi(z)=\psi(h(z))^{2}(u(z)-\epsilon)$ for $z \in U$ and $\phi \equiv 0$ for $z \in D \backslash U$. Using [9, Theorem 1.20] we conclude that $\phi \in A C L_{\text {loc }}^{2}(D)$. Because $\operatorname{supp} \phi \subset \subset D$ we have by Green's formula

$$
\int_{U} d(\phi d v)=0
$$

and hence

$$
\int_{U} d \phi \wedge d v=0
$$

Because

$$
d \phi \wedge d v=\psi^{2} d u \wedge d v+2 \psi(u-\epsilon) d \psi \wedge d v
$$

we obtain

$$
\int_{U} \psi^{2}\left(u_{x} v_{y}-u_{y} v_{x}\right) d x d y=-2 \int_{U} \psi(u-\epsilon)\left(\psi_{x} v_{y}-\psi_{y} v_{x}\right) d x d y .
$$

This is clear for $u, v \in C^{2}(D)$. In the general case we can easily prove it if we use a standard approximation procedure (see, for example, [9, Lemma 14.15]).

From here

$$
\int_{U} \psi^{2}\left(u_{x} v_{y}-u_{y} v_{x}\right) d x d y \leq 2 \int_{U} \psi|u-\epsilon||\nabla \psi||\nabla v| d x d y .
$$


Using (1.1) we find

$$
\begin{aligned}
& \int_{U} \psi^{2}\left(a u_{x}^{2}+(b+c) u_{x} u_{y}+d u_{y}^{2}\right) d x d y \\
& \leq 4 M \int_{U} \psi|\nabla \psi| \sqrt{\left(a u_{x}+b u_{y}\right)^{2}+\left(c u_{x}+d u_{y}\right)^{2}} d x d y \\
& =4 M \int_{U} \psi|\nabla \psi| \sqrt{\left(a^{2}+c^{2}\right) u_{x}^{2}+2(a b+c d) u_{x} u_{y}+\left(b^{2}+d^{2}\right) u_{y}^{2}} d x d y \\
& \leq 4 M\left(\int_{U}|\nabla \psi|^{2} \frac{\left(a^{2}+c^{2}\right) u_{x}^{2}+2(a b+c d) u_{x} u_{y}+\left(b^{2}+d^{2}\right) u_{y}^{2}}{a u_{x}^{2}+(b+c) u_{x} u_{y}+d u_{y}^{2}} d x d y\right)^{1 / 2} \\
& \times\left(\int_{U} \psi^{2}\left(a u_{x}^{2}+(b+c) u_{x} u_{y}+d u_{y}^{2}\right) d x d y\right)^{1 / 2} .
\end{aligned}
$$

Thus we obtain

$$
\begin{aligned}
& \int_{U} \psi^{2}\left(a u_{x}^{2}+(b+c) u_{x} u_{y}+d u_{y}^{2}\right) d x d y \\
\leq & 16 M^{2} \int_{U}|\nabla \psi|^{2} \frac{\left(a^{2}+c^{2}\right) u_{x}^{2}+2(a b+c d) u_{x} u_{y}+\left(b^{2}+d^{2}\right) u_{y}^{2}}{a u_{x}^{2}+(b+c) u_{x} u_{y}+d u_{y}^{2}} d x d y .
\end{aligned}
$$

We write

$$
I(\xi, \eta)=\left(a^{2}+c^{2}\right) \xi^{2}+2(a b+c d) \xi \eta+\left(b^{2}+d^{2}\right) \eta^{2}
$$

and

$$
I I(\xi, \eta)=\left(a \xi^{2}+(b+c) \xi \eta+d \eta^{2}\right) .
$$

From (1.2) it follows that the quadratic form $I I(\xi, \eta)$ is positive definite, and hence the bundle of quadratic forms

$$
H(\xi, \eta ; \lambda)=I(\xi, \eta)-\lambda I I(\xi, \eta)
$$

is regular in the sense of [7, Chapter $\mathrm{X}]$.

Let $\lambda_{\max }$ be a maximal eigenvalue of $H(\xi, \eta ; \lambda)$. Then using well-known properties of quadratic forms (see [7, Theorem 13, Chapter X]) for every $\xi, \eta \neq 0$, we have

$$
\frac{I(\xi, \eta)}{I I(\xi, \eta)} \leq \lambda_{\max }
$$

The characteristic equation of $H(\xi, \eta ; \lambda)$ has the form

i.e.,

$$
\begin{array}{ll}
a^{2}+c^{2}-\lambda a & a b+c d-\lambda(b+c) / 2 \\
a b+c d-\lambda(b+c) / 2 & b^{2}+d^{2}-\lambda d
\end{array} \mid=0
$$

$$
\delta \lambda^{2}-(a+d)(a d-b c) \lambda+(a d-b c)^{2}=0 .
$$


Solving this equation for $\lambda$ and using (1.2) we find that

$$
\lambda_{\max } \leq \mu \equiv \frac{(a d-b c)(a+d)}{\delta} .
$$

Clearly, $a d-b c>0$ since from (1.2) it follows that

$$
0<4 a d-(b+c)^{2} \leq 4 a d-4 b c .
$$

By (2.3) for every $(x, y) \in U$, we obtain

$$
\frac{\left(a^{2}+c^{2}\right) u_{x}^{2}+2(a b+c d) u_{x} u_{y}+\left(b^{2}+d^{2}\right) u_{y}^{2}}{a u_{x}^{2}+(b+c) u_{x} u_{y}+d u_{y}^{2}} \leq \mu .
$$

Thus from (2.2) we arrive at the estimate

$$
\int_{U} \psi^{2}\left(a u_{x}^{2}+(b+c) u_{x} u_{y}+d u_{y}^{2}\right) d x d y \leq 16 M^{2} \int_{U} \mu|\nabla \phi|^{2} d x d y .
$$

Now we shall estimate the integral in the right side of (2.5). We have

$$
I \equiv \int_{U} \mu|\nabla \phi|^{2} d x d y=\int_{\substack{\delta^{\prime}<h(z)<\delta^{\prime \prime} \\ z \in U}} \mu \psi_{0}^{\prime 2}(h(z))|\nabla h|^{2} d x d y
$$

and next, by the well-known Kronrod-Federer coarea formula [3, Chapter $3, \S \mathbf{2 . 4}$ ],

$$
I=\int_{\delta^{\prime}}^{\delta^{\prime \prime}} \psi_{0}^{\prime 2}(t) d t \int_{E_{t} \cap U} \mu|\nabla h| d \mathcal{H}^{1}\left(E_{t}\right) .
$$

Using (1.5) we find

$$
I \leq h_{1} \int_{\delta^{\prime}}^{\delta^{\prime \prime}} \psi_{0}^{\prime 2}(t) d t \int_{E_{t} \cap U} \mu d \mathcal{H}^{1}\left(E_{t}\right) .
$$

Since $\psi_{0}\left(\delta^{\prime}\right)=1$ and $\psi_{0}\left(\delta^{\prime \prime}\right)=0$, we have

$$
\begin{aligned}
1 \leq\left(\int_{\delta^{\prime}}^{\delta^{\prime \prime}}\left|\psi_{0}^{\prime}(t)\right| d t\right)^{2} & \leq \int_{\delta^{\prime}}^{\delta^{\prime \prime}}\left|\psi_{0}^{\prime}\right|^{2} d t \int_{E_{t} \cap U} \mu d \mathcal{H}^{1}\left(E_{t}\right) \\
& \times \int_{\delta^{\prime}}^{\delta^{\prime \prime}} d t\left(\int_{E_{t} \cap U} \mu d \mathcal{H}^{1}\left(E_{t}\right)\right)^{-1} .
\end{aligned}
$$

Therefore, for every absolutely continuous function $\psi_{0}:\left[\delta^{\prime}, \delta^{\prime \prime}\right] \rightarrow[0,1]$ such that $\psi_{0}\left(\delta^{\prime \prime}\right)=1$ and $\psi_{0}\left(\delta^{\prime}\right)=0$, we have

$$
\left(\int_{\delta^{\prime}}^{\delta^{\prime \prime}} d t\left(\int_{E_{t} \cap U} \mu d \mathcal{H}^{1}\left(E_{t}\right)\right)^{-1}\right)^{-1} \leq \int_{\delta^{\prime}}^{\delta^{\prime \prime}}\left|\psi_{0}^{\prime}\right|^{2} d t \int_{E_{t} \cap U} \mu d \mathcal{H}^{1}\left(E_{t}\right) .
$$


Choosing for $\delta^{\prime} \leq \tau \leq \delta^{\prime \prime}$,

$$
\psi_{0}(\tau)=\int_{\delta^{\prime}}^{\tau} d t\left(\int_{E_{t} \cap U} \mu d \mathcal{H}^{1}\left(E_{t}\right)\right)^{-1} / \int_{\delta^{\prime}}^{\delta^{\prime \prime}} d t\left(\int_{E_{t} \cap U} \mu d \mathcal{H}^{1}\left(E_{t}\right)\right)^{-1},
$$

we have

$$
\min _{\psi_{0}} \int_{\delta^{\prime}}^{\delta^{\prime \prime}}\left|\psi_{0}^{\prime}\right|^{2} d t \int_{E_{t} \cap U} \mu d \mathcal{H}^{1}\left(E_{t}\right)=\left(\int_{\delta^{\prime}}^{\delta^{\prime \prime}} d t\left(\int_{E_{t} \cap U} \mu d \mathcal{H}^{1}\left(E_{t}\right)\right)^{-1}\right)^{-1} .
$$

Thus from (2.5) and (2.6) we see that

$$
\begin{aligned}
& \int_{\substack{\delta^{\prime \prime}<h(z) \\
z \in U}}\left(a u_{x}^{2}+(b+c) u_{x} u_{y}+d u_{y}^{2}\right) d x d y \\
& \leq 16 M^{2} h_{1}\left(\int_{\delta^{\prime}}^{\delta^{\prime \prime}} d t\left(\int_{E_{t} \cap U} \mu d \mathcal{H}^{1}\left(E_{t}\right)\right)^{-1}\right)^{-1} .
\end{aligned}
$$

Setting here $\delta^{\prime} \rightarrow 0$ we obtain

$$
\begin{aligned}
& \int_{\substack{\delta^{\prime \prime}<h(z) \\
z \in U}}\left(a u_{x}^{2}+(b+c) u_{x} u_{y}+d u_{y}^{2}\right) d x d y \\
& \leq 16 M^{2} h_{1}\left(\int_{0}^{\delta^{\prime \prime}} d t\left(\int_{E_{t} \cap U} \mu d \mathcal{H}^{1}\left(E_{t}\right)\right)^{-1}\right)^{-1} .
\end{aligned}
$$

Previously we chose $\delta^{\prime \prime}<h\left(z_{0}\right)$ where $z_{0} \in U$. Thus the open set $U_{1}=\{z \in U$ : $\left.\delta^{\prime \prime}<h\left(z_{0}\right)\right\}$ is not empty. Now from (1.7) we conclude that

$$
\int_{U_{1}}\left(a u_{x}^{2}+(b+c) u_{x} u_{y}+d u_{y}^{2}\right) d x d y=0,
$$

that is,

$$
a u_{x}^{2}+(b+c) u_{x} u_{y}+d u_{y}^{2}=0 \quad \text { a.e. in } U_{1} .
$$

The assumption (1.2) implies that $\nabla u=0$ a.e. in $U_{1}$ and hence $u \equiv$ const in $U_{1}$. But $\delta^{\prime \prime}>\delta^{\prime}>0$ can be arbitrarily small, and hence $\nabla u=0$ a.e. in $U$. There is a contradiction with the definition of the connected component $U$ and (2.1) is true.

Analogously, we obtain

$$
\inf _{\Delta} u(z)=\inf _{\partial^{\prime} \Delta} u(z)
$$

and next, we arrive at (1.4).

\section{Proof of Theorem 1.8}

Suppose that there exists a homeomorphic solution $w(z)$ of (1.1), (1.2) such that $w(D)=\mathcal{D}$ and $L \subset w(\Gamma)$. Since $L$ is $u$-forked, it follows that there is a vertical segment $l \subset \mathcal{D}$ with the end points in $L$ and moreover, there exists a subdomain 
$U$ of $\mathcal{D}$ with the boundary $\tilde{\partial} U \subset \bar{l} \cup L$. Consider its preimage $\Delta=w^{-1}(U)$. By Theorem 1.6 the function $u$ is monotone close to $\Gamma$. Hence,

$$
\begin{aligned}
\operatorname{osc}(u, \Delta) & \leq \operatorname{osc}\left(u, \tilde{\partial}^{\prime} \Delta\right) \\
& =\operatorname{osc}\left(u, w^{-1}(l)\right)=0 .
\end{aligned}
$$

Thus, $u \equiv$ const in $\Delta$, which is impossible.

\section{An example}

Let $\gamma$ be a simple open Jordan arc lying in the upper half-plane with end points $(0,0)$ and $(1,0)$ on the $x$-axis. We set

$$
\Gamma=\{(x, y): 0 \leq x \leq 1, y=0\},
$$

and denote by $D$ the subdomain of $\mathbf{R}^{2}$ enclosed by $\gamma \cup \Gamma$.

Choose in Theorem 1.8 the function $h(z)=y$. Evidently, (1.5) is fulfilled.

Consider the case of (1.1) in which the functions $a=a(y), d=d(y)$ are positive and $b=c=0$. Then (1.1) admits the form

$$
a(y) u_{x}=v_{y}, \quad d(y) u_{y}=-v_{x} .
$$

The assumption (1.2) is fulfilled too.

The assumption (1.7) takes the form

$$
\int_{0} \frac{d y}{a(y)+d(y)}=\infty
$$

We obtain:

Corollary 4.3. Let $\mathcal{D}$ be a simply connected subdomain of $\{(u, v):|u|<M\}$, where $0<M<\infty$ is a constant. Assume that (4.2) is fulfilled and $L \subset \tilde{\partial} \mathcal{D}$ is u-forked. Then there are no $A C L_{\text {loc }}^{2}$ solutions $w=w(z)$ of 4.1 mapping $D$ homeomorphically onto $\mathcal{D}$ such that $L \subset w(\Gamma)$.

\section{ACKNOWLEDGements}

The authors are indebted to Prof. V.A. Klyachin and to the anonymous referee for a number of useful remarks and corrections.

\section{REFERENCES}

[1] B.V. Bojarski: Generalized solutions of an elliptic system of first order with discontinuous coefficients (in Russian), Mat. Sb. 43, No. 4, 451-503, 1957. MR0106324 (21:5058)

[2] M.A. Brakalova And J.A. Jenkins: On solutions of the Beltrami equation. J. Anal. Math. 76 (1998), 67-92. MR:1676936|(2000h:30029)

[3] Yu. Burago and V. A. Zalgaller: Geometric inequalities, Leningrad, "Nauka", 1980 (in Russian). MR0602952 (82d:52009)

[4] E.A. Chtcherbakov (Ś̌ERbaKov): Homeomorphic solutions of degenerate elliptic systems (in Russian), Math. Phys., Kiev, Naukova Dumka 8, 187-190, 1970. MR0293239 $(45: 2316)$

[5] G. DAvid: Solutions de l'équation de Beltrami avec $\|\mu\|_{\infty}=1$, Ann. Acad. Sci. Fenn. Ser. A I Math. 13, 25-70, 1988. MR0975566 (90d:30058)

[6] A. Džuraev: On a Beltrami system of equations degenerating on a line, - Soviet Math. Dokl. 10, No. 2, 1969, 449-452. (Russian) Dokl. Akad. Nauk SSSR 185 1969, 984-986. MR0241668 (39:3007) 
[7] F.R. Gantmacher: Theory of Matrices, Chelsea, New York, 1959. MR0107649(21:6372c)

[8] V. Gutlyanskit, O. Martio, T. Sugawa and M. Vuorinen: On the degenerate Beltrami equation, Reports of the Dept. of Math., Univ. of Helsinki, Preprint 282, 2001, 1-32, Trans. Amer. Math. Soc. (to appear).

[9] J. Heinonen, T. Kilpeläinen and O. Martio: Nonlinear Potential Theory of Degenerate Elliptic Equations, Oxford Mathematical Monographs, Oxford University Press, Oxford, 1993. MR1207810 (94e:31003)

[10] O. Martio And V.M. Miklyukov: On existence and uniqueness of degenerate Beltrami equation, Reports of the Dept. of Math., Univ. of Helsinki, Preprint 347, 2003, 1-12.

[11] O. Martio, V.M. Miklyukov and M. Vuorinen: Functions monotone close to boundary, Reports of the Dept. of Math., Univ. of Helsinki, Preprint 330, March 2002, 1-20, Tohoku Math. J. (to appear).

[12] A.P. Minailov: A problem for a mapping by solutions of elliptic system degenerating on boundary (in Russian), Sibirsk. Mat. Zh. 24, No. 3, 119-127, 1983. MR0704163 (84m:30075)

[13] I.S. OvChinNikov: On existence of plane mappings for degenerate elliptic systems of the first order (in Russian), Dokl. AN SSSR 191, No. 3, 526-529, 1970. MR0259355 (41:3993)

[14] U. SRebro And E. Yakubov: Branched folder maps and alternating Beltrami equations, J. Anal. Math. 70, 65-90, 1996. MR1444258 (98f:30021)

[15] W.P. Ziemer: Weakly Differentiable Functions, Graduate Texts in Mathematics 120, Springer-Verlag, New York, 1989. MR1014685 (91e:46046)

Department of Mathematics and Statistics, P.O. Box 68, Fin-00014, University of HELSINKI, FinLAND

E-mail address: martio@cc.helsinki.fi

Department of Mathematics, Volgograd State University, 2 Prodolnaya, 30, VolGOGRAD, 400062, RUSSIA

E-mail address: miklyuk@mail.ru

Department of Mathematics, Fin-20014, University of Turku, Finland

E-mail address: vuorinen@csc.fi 